\title{
Validation of a registry-derived risk algorithm based on treatment protocol as a proxy for disease risk in childhood acute lymphoblastic leukemia
}

\author{
Sumit Gupta ${ }^{1,5^{*}}$, Jason D Pole ${ }^{2,3}$, Astrid Guttmann ${ }^{3,4,5}$ and Lillian Sung ${ }^{1,5}$
}

\begin{abstract}
Background: Administrative databases and cancer registries are frequently used to conduct population-based research, but often lack clinical data necessary for risk stratification. Our objective was to determine the criterion validity of a risk-stratification algorithm based on treatment characteristics available from a pediatric cancer registry as a proxy for disease risk, by comparing it to traditional biology-based risk classifications.

Methods: We identified all children with acute lymphoblastic leukemia diagnosed at a single institution between January 2000 and June 2011, and linked them to a population-based cancer registry. Several risk algorithms were then constructed using disease risk variables collected through chart review by a pediatric oncologist, and compared to a risk algorithm based on treatment protocol name and age, available from the registry.

Results: Of 596 patients identified, 579 (97.1\%) met inclusion criteria and were successfully linked. The registry-based algorithm showed almost perfect agreement with a biology-based algorithm based on age, initial white blood cell count, immunophenotype and cytogenetics (kappa $=0.85,95^{\text {th }}$ confidence interval 0.81-0.90). Discrepant cases were often due to the presence of unusual high risk features not captured by standard disease-risk variables but reflected in clinicians' choices of higher intensity treatment protocols.

Conclusions: Protocol name represents a valid proxy of disease risk, allowing for risk stratification while conducting comparative effectiveness research using cancer registries and health services data. Future studies should examine the validity of treatment-based risk algorithms in other malignancies and using other treatment characteristics commonly found in health services data, such as the receipt of specific chemotherapeutic agents.
\end{abstract}

Keywords: Acute lymphoblastic leukemia, Administrative data, Health services research, Registries, Validation

\section{Background}

The past several decades have witnessed remarkable advances in the treatment of childhood cancer, with overall cure rates now exceeding $80 \%$ [1-3]. However, in many cases significant differences exist between outcomes reported from clinical trials and population-based data. For example, Hunger et al. recently reported that children over the age of 15 years with acute lymphoblastic leukemia

\footnotetext{
* Correspondence: sumit.gupta@sickkids.ca

${ }^{1}$ Division of Haematology/Oncology and Program in Child Health Evaluative Sciences, Hospital for Sick Children, 555 University Avenue, Toronto, Ontario, Canada M5G 1 X8

${ }^{5}$ Department of Paediatrics and Institute for Health, Policy Management and Evaluation, University of Toronto, 155 College Street, Suite 425, Toronto,

Ontario, Canada M5T 3M6

Full list of author information is available at the end of the article
}

(ALL) treated on Children's Oncology Group clinical trials between 2000-2005 had a 5-year survival rate of 76\% [4]. By contrast, registry data for 15-19 year olds diagnosed with ALL over a similar time period showed a far lower 5 -year survival of 50.1\% [3]. In addition to allowing better capture of population survival trends, cancer registries and health services databases have also been used in pediatric oncology to conduct comparative effectiveness research, identify survivors at high risk of long term medical and socioeconomic adverse effects, and monitor the uptake of new therapeutic interventions [5-9].

While routinely collected population-based data holds significant potential, it can also introduce new biases. One major limitation in many of these datasets is the inability to risk stratify patients. In childhood cancer, detailed

\section{Biomed Central}


staging, histologic, genetic and response-based information is used to determine the risk of mortality and other adverse outcomes; prognosis and treatment can vary widely within a single malignancy based on this information $[10,11]$. These detailed biologic data are rarely collected by cancer registries or administrative databases, as highlighted by a recent review of data sources for cancer comparative effectiveness research [12]. Thus important potential confounding information is often unavailable, limiting confidence in the conclusions of studies using these resources.

A valid method of risk stratification using information available in population-based databases would increase the contribution of these data. Treatment-based risk assignment may offer such a method. In pediatric cancer, treatment intensity is often based on disease risk and biologic prognostic factors; high-risk subtypes of a particular malignancy will receive higher intensity treatment $[10,11,13]$. Treatment information is often collected in population-based databases: cancer registries may collect the names of treatment protocols while health services databases may collect information on the administration of particular chemotherapeutic agents [8,14].

Our objective was therefore to determine the criterion validity of a registry-based risk-stratification algorithm using treatment protocol name and age by comparing it to several traditional biology-based risk classifications. We undertook this in a single-institution cohort of children with ALL.

\section{Methods}

\section{Study population}

The study population included all children diagnosed with primary ALL between June 1, 2000 and December 31, 2011 at The Hospital for Sick Children, Toronto, Canada. The Hospital for Sick Children is a pediatric tertiary care institution that sees over 300 new cases of childhood cancer per year. Non-Ontario residents, children for whom no active treatment was pursued, and children transferred to other centers within the first month of treatment were excluded. Patients were identified using a local institutional electronic database. ALL was chosen as it has one of the most refined risk determination classifications in pediatric oncology, incorporating multiple biologic factors [10].

\section{Data sources and variables}

Factors collected for each patient by chart review were: age at diagnosis, initial white blood cells (WBC) at diagnosis, immunophenotype/lineage, leukemia cytogenetics, and the presence of minimally residual disease (MRD) at the end of induction therapy. Cytogenetic abnormalities considered high risk included $t(9 ; 22)$ (BCR-ABL), hypodiploidy ( $<45$ chromosomes), and any
$11 \mathrm{q} 23$ (MLL) rearrangement $[15,16]$. MRD was assessed by flow cytometry; $\geq 0.01 \%$ residual blasts in bone marrow at the end of induction was considered positive [17]. These variables were chosen a priori based on both their accepted use in contemporary ALL risk stratification and the ease of their availability in patient charts [10].

POGONIS is a population-based registry that prospectively captures all cases of pediatric cancer diagnosed and treated at one of the five tertiary pediatric oncology centers in Ontario. POGONIS personnel assign each patient a unique numeric identifier, which is retained both by the treating centers and POGONIS. This number was therefore used to link study patients to POGONIS. Approximately $98 \%$ of Ontario children with cancer aged $0-14$, as identified by the Ontario Cancer Registry (OCR), are captured in POGONIS [14]. Basic demographic, treatment and outcome variables are available in POGONIS. Though various biologic fields have been introduced into POGONIS at different times, they are variably collected between centers and often incompletely available. Therefore only treatment protocol and age at diagnosis were utilized from POGONIS for this study.

Multiple protocols for an individual child could be listed in POGONIS if clinicians changed treatment based on toxicity, non-response, or new prognostic information. Any subsequent protocol recorded within four weeks of the start of the first protocol was therefore identified and compared to the first. In such cases, the more specific protocol was ultimately assigned to the patient. For example, the specific protocol "AALL0331" was chosen over the more generic "three drug induction". Where two specific protocols were identified, the one with a later start date was chosen in order to better reflect risk prognosticators available after diagnosis.

A pediatric oncologist identified patients, determined eligibility and conducted the chart abstraction for all patients. At the time of chart review, the abstractor was blinded to all patient registry data.

\section{Creation of risk algorithms}

Biology-based risk algorithms were created using variables abstracted by chart review while a registry-based risk algorithm was created using treatment protocol name listed in POGONIS and age at diagnosis. Multiple biology-based risk algorithms were constructed using these data to divide patients into standard and high risk strata. Within each of these algorithms, the presence of any high risk feature resulted in classification in the high risk stratum. The first algorithm utilized only age and presenting WBC according to the National Cancer Institution/Rome criteria (standard risk $=$ age $\geq 1$ year and $<10$ years, and WBC $<50 \times 10^{9} / \mathrm{L}$ ) [18]. Subsequent algorithms added additional prognosticators in order to produce sequentially more sophisticated risk classifications using the 
following high risk classifiers: $\mathrm{T}$ cell immunophenotype, poor risk cytogenetics, and presence of MRD (Table 1). Where a prognosticator was not available for a particular patient, the algorithm treated it as non-informative.

All treatment protocols used during the study period were a priori classified as standard or high risk based on protocol inclusion and exclusion criteria (Table 2). Protocol inclusion criteria tend to mirror contemporaneous knowledge of favorable and unfavorable risk factors. Thus the inclusion criteria of past protocols do not perfectly align with modern definitions of standard or high risk. All protocols were therefore classified according to whether treating physicians at the time would have considered them standard or high risk. All protocol risk assignments were made through the consensus of two pediatric oncologists.

Age and protocol risk classification were then used to create a registry-based risk algorithm. Patients aged 1-9 years at diagnosis and treated on a protocol classified as standard risk were designated as standard risk. Patients $<1$ year at diagnosis, $\geq 10$ years at diagnosis or treated on a protocol classified as high risk were designated as high risk.

All biology-based and registry-based risk algorithms were created prior to the collection of any patient data.

\section{Analysis}

The agreement between the registry-based risk algorithm and each of the biology-based risk algorithms was assessed using the kappa statistic (0.00-0.20 slight; 0.21-0.40 fair; 0.41-0.60 moderate; 0.61-0.80 substantial; 0.81-1.00 almost perfect) [19]. The charts of discrepant cases were reviewed in detail in order to identify possible reasons for discordance. Statistical analyses were performed using SAS-PC software (version 9.2; SAS Institute, Cary, NC). Ethics approval was obtained from The Hospital for Sick Children.

\section{Results}

A total of 596 patients were diagnosed during the study period. Twelve children were excluded: seven were not Ontario residents, three were transferred to other centers within a week of starting treatment, one represented a second malignancy, and one had no treatment pursued due to underlying comorbidities. Of the remaining 584, 579 (99.1\%) were successfully linked to POGONIS. All five unlinked patients were diagnosed in the last 21 days of 2011 and had therefore not yet been registered in POGONIS at the time of data analysis.

The median age of the cohort was 4 years (interquartile range 3-8). A total of 343 (59\%) patients were male. Disease-related characteristics of the cohort can be seen in Table 3. MRD results were only available on approximately half of the cohort as this investigation was only introduced into routine practice in 2005. The vast majority of patients were treated with defined protocols; treatment protocols were assigned for all but four of the study patients $(575 / 579 ; 99.3 \%)$. Two patients died prior to or shortly after starting treatment, while no reason could be identified for the lack of listed protocol in the remaining two. The most commonly used protocols were from the Children's Oncology Group: AALL0331 and AALL0232, accounting for 184 (31.8\%) and 107 (18.48\%) of patients respectively.

The number of children classified as standard risk by the biology-based algorithms varied from 62.0\% (algorithm 1 [simplest] - age and WBC) to 56.0\% (algorithm 4 [most complex] - incorporating all factors of age, WBC, immunophenotype, cytogenetics and MRD) (Table 3). The registry-based algorithm classified $56.7 \%$ of patients as standard risk. Table 3 also illustrates the distribution of each disease characteristic by risk category, and its variation across algorithms.

Table 4 shows the agreement between the registrybased classification and the biology-based algorithms. Agreement was excellent $(k \geq 0.80)$ in all cases $[19,20]$. The best agreement as judged by the kappa statistic was between the registry-based algorithm and algorithms 2 (age, WBC, and immunophenotype) and 3 (age, WBC, immunophenotype and cytogenetics).

A total of 14 patients were classified as standard risk by the registry-based algorithm but as high risk by the biology-based algorithm 3. In two cases, this was due to data entry errors within POGONIS. In the remaining 12 cases, the misclassification was due to disease features currently known to be adverse prognosticators but

Table 1 Biology-based risk algorithms using data available from chart review

\begin{tabular}{|c|c|c|c|c|c|c|c|c|c|c|}
\hline & \multicolumn{2}{|c|}{ Age (years) } & \multicolumn{2}{|c|}{ WBC $\left(\times 10^{9} / \mathrm{L}\right)$} & \multicolumn{2}{|c|}{ Immunophenotype } & \multicolumn{2}{|c|}{ Cytogenetics $^{\&}$} & \multicolumn{2}{|c|}{$\mathrm{MRD}^{\#}$} \\
\hline & $1-9$ & $<1, \geq 10$ & $<50$ & $\geq 50$ & B & $T$ & Low risk & High risk & Negative & Positive \\
\hline Algorithm $1^{*}$ & SR & $\mathrm{HR}$ & SR & $\mathrm{HR}$ & - & - & - & - & - & - \\
\hline Algorithm $2^{*}$ & SR & $H R$ & $\mathrm{SR}$ & $H R$ & SR & $H R$ & - & - & - & - \\
\hline Algorithm $3^{*}$ & SR & $H R$ & SR & $H R$ & SR & $H R$ & SR & $H R$ & - & - \\
\hline Algorithm $4^{*}$ & $S R$ & $H R$ & SR & $\mathrm{HR}$ & SR & $H R$ & $S R$ & $H R$ & SR & $H R$ \\
\hline
\end{tabular}

$H R$ High risk, MRD Minimally residual disease, SR Standard risk, WBC White blood cell.

*For each algorithm, patients were classified as standard risk only in the absence of any high risk feature.

\&High risk cytogenetics included t(9;22) (BCR-ABL), hypodiploidy ( $<45$ chromosomes), or any 11 q23 (MLL) rearrangement.

\#MRD positivity was defined as $\geq 0.01$ residual blasts. 
Table 2 Registry-based algorithm based on treatment protocol name and details of each treatment protocol

\begin{tabular}{|c|c|c|c|c|c|c|}
\hline Protocol & Lineage & Age (years) & WBC $\left(x 10^{9} / \mathrm{L}\right)$ & Genetics & Other & Risk assignment \\
\hline AALL02P2 & $\mathrm{T}$ & $\geq 10$ (or) $^{*}$ & $\geq 50$ (or) & & & $\mathrm{HR}$ \\
\hline AALL0031 & & & & $\begin{array}{l}t(9 ; 22) \text { or hypodiploidy or MLL } \\
\text { with slow response (or) }\end{array}$ & Induction failure (or) & $\mathrm{HR}$ \\
\hline AALL0232 & B & $\geq 10$ (or) & $\geq 50$ (or) & & Steroid pre treatment (or) & $\mathrm{HR}$ \\
\hline AALL0331 & B & $1-9$ & $<50$ & & & SR \\
\hline AALL0434 & $\mathrm{T}$ & $>1$ & & & & $H R$ \\
\hline AALL0622 & & $>1$ & & $\mathrm{t}(9 ; 22)$ & & $\mathrm{HR}$ \\
\hline AALL0631 & & $<1$ & & & & $H R$ \\
\hline AALL0932 & B & $1-9$ & $<50$ & $\begin{array}{l}\text { No t(9;22), MLL, iAMP21, } \\
\text { hypodiploidy }\end{array}$ & & SR \\
\hline CCG1991 & B & $1-9$ & $<50$ & & & SR \\
\hline POG9201 & B & $1-9$ & $<50$ & $\begin{array}{c}\text { Trisomy } 4,10, \mathrm{Dl}>1.16 \text { or } \mathrm{t}(12 ; 21) \\
\text { No } \mathrm{t}(9 ; 22), 1 ; 19, \mathrm{MLL}\end{array}$ & & SR \\
\hline POG9407 & & $<1$ & & & & $H R$ \\
\hline \multirow[t]{3}{*}{ POG9605 } & B & $1-9$ & $<50$ & $\begin{array}{c}\text { No trisomy } 4,10, \mathrm{Dl}>1.16, \mathrm{MLL}, \\
\mathrm{t}(1 ; 19), \mathrm{t}(9 ; 22)\end{array}$ & & SR \\
\hline & B & $\geq 10$ & $<50$ & $\begin{array}{c}\text { Trisomy 4,10 or Dl>1.16; No MLL, } \\
\mathrm{t}(1 ; 19), \mathrm{t}(9 ; 22)\end{array}$ & & SR \\
\hline & B & $>1$ & $\geq 50$ & $\begin{array}{c}\text { Trisomy 4,10 or Dl>1.16; No MLL, } \\
\text { t(1;19), t(9;22) }\end{array}$ & & SR \\
\hline POG9904 & B & $1-9$ & $<50$ & $\begin{array}{c}\text { Trisomy } 4,10, \mathrm{Dl}>1.16 \text { or } \mathrm{t}(12 ; 21) \\
\text { No } \mathrm{MLL}, \mathrm{t}(1 ; 19), \mathrm{t}(9 ; 22)\end{array}$ & & SR \\
\hline POG9905 & & & & & Neither 9904, 9906 nor AALL0031 & SR \\
\hline \multirow[t]{2}{*}{ POG9906 } & B & $M>12 ; F>16$ (or) & $>100$ (or) & MLL (or) & & $H R$ \\
\hline & B & \multicolumn{3}{|c|}{ Sliding scale of WBC criteria for $\mathrm{M}$ age 8-11 and $\mathrm{F}$ age 12-15 } & & $H R$ \\
\hline Protocol C & & $<1$ or $>10$ (or) & $>20$ (or) & $\mathrm{t}(9 ; 22)$ or MLL (or) & $\begin{array}{l}\text { L2 morphology, mediastinal mass, } \\
\text { or massive LN/HSM }\end{array}$ & $H R$ \\
\hline
\end{tabular}

DI DNA index, F Female, HR High risk, HSM Hepatosplenomegaly, iAMP Intra-amplification, LN - Lymphadenopathy, M Male, SR Standard risk, WBC White blood cell. *(or) indicates factors for which any one was sufficient for inclusion into the protocol.

which were not considered so at the time of the patient's presentation (e.g. specific high-risk cytogenetic abnormalities). A further 27 patients were classified as high risk by the registry-based algorithm and as standard risk by the biology-based algorithm 3. Seven cases were due to data entry errors, predominantly in protocol name. Twelve cases were due to the presence of risk factors other than the a priori specified biology-based factors obtained by chart review, such as central nervous system involvement, testicular involvement, and steroid pre-treatment. In the remaining cases, clinicians chose high-risk treatment protocols for individualized reasons despite patients meeting standard risk criteria. A common example among these remaining cases involved the WBC count at diagnosis: several children presented with counts of $<50 \times 10^{9} / \mathrm{L}$ (thus meeting standard risk criteria), but which then shortly rose to $\geq 50 \times 10^{9} / \mathrm{L}$ prior to the start of therapy.

\section{Discussion}

We found that for children with ALL, a risk stratification system based on treatment protocol as recorded in a population-based pediatric cancer registry was a valid proxy of disease risk as compared to biology-based risk algorithms. Agreement between the registry-based algorithm and the biology-based algorithm incorporating age, WBC, immunophenotype and cytogenetics was almost perfect, with a kappa of 0.85 (95\% confidence interval 0.81 to 0.90 ).

Though still almost perfect, agreement between the registry-based algorithm was lower with algorithm 1 (age, WBC; $k=0.80$ ) and with algorithm 4 (all biologic prognosticators, including MRD; $k=0.83$ ). The registry-based classification was likely superior to the simplest algorithm incorporating only age and initial WBC (algorithm 1), as a portion of children considered standard risk by age and WBC will in fact have high risk immunophenotypic or cytogenetic features (Table 3); this was reflected in the treatment given to them. By contrast, as MRD results are only available approximately five weeks after starting treatment, it is unlikely that our protocol-based algorithm as currently defined captured MRD-based changes in treatment. However, high risk cytogenetics and MRD positivity 
Table 3 Disease-related characteristics of overall study cohort and distribution of each characteristic by biology-based algorithms $(\mathrm{N}=579)$

\begin{tabular}{|c|c|c|c|c|c|c|c|c|c|}
\hline & \multirow[b]{3}{*}{ Overall } & \multirow{2}{*}{\multicolumn{2}{|c|}{$\begin{array}{c}\text { Algorithm } 1^{*} \\
\text { Age, WBC }\end{array}$}} & \multicolumn{2}{|c|}{ Algorithm 2* } & \multicolumn{2}{|c|}{ Algorithm 3* } & \multirow{2}{*}{\multicolumn{2}{|c|}{$\frac{\text { Algorithm } 4^{*}}{\text { Algorithm 3+MRD }}$}} \\
\hline & & & & \multicolumn{2}{|c|}{ Algorithm 1+ immunophenotype } & \multicolumn{2}{|c|}{ Algorithm 2+cytogenetics } & & \\
\hline & & SR & HR & SR & HR & SR & HR & SR & HR \\
\hline Overall & $579(100)$ & $359(62)$ & $220(38)$ & $347(60)$ & $232(40)$ & $340(59)$ & $239(41)$ & $324(56)$ & $255(44)$ \\
\hline Immunophenotype & & & & & & & & & \\
\hline B & $522(90)$ & $347(66)$ & $175(34)$ & $347(66)$ & $175(34)$ & $340(65)$ & $182(35)$ & $324(62)$ & $198(10)$ \\
\hline T & $57(10)$ & $12(21)$ & $45(79)$ & $0(0)$ & $57(100)$ & $0(0)$ & $57(100)$ & $0(0)$ & $57(100)$ \\
\hline Cytogenetics & & & & & & & & & \\
\hline High risk & $36(6)$ & $7(19)$ & $29(81)$ & $7(19)$ & $29(81)$ & $0(0)$ & $36(100)$ & $0(0)$ & $36(100)$ \\
\hline MLL rearrangement & $19(3)$ & $3(16)$ & $16(84)$ & $3(16)$ & $16(84)$ & $0(0)$ & $19(100)$ & $0(0)$ & $19(100)$ \\
\hline $\mathrm{t}(9 ; 22)(\mathrm{BCR}-\mathrm{ABL})$ & $10(2)$ & $2(20)$ & $8(80)$ & $2(20)$ & $8(80)$ & $0(0)$ & $10(100)$ & $0(0)$ & $10(100)$ \\
\hline Hypodiploidy & $7(1)$ & $2(29)$ & $5(71)$ & $2(29)$ & $5(71)$ & $0(0)$ & $7(100)$ & $0(0)$ & $7(100)$ \\
\hline Standard risk & $542(94)$ & $352(65)$ & $190(35)$ & $340(63)$ & $202(37)$ & $340(63)$ & $202(37)$ & $324(60)$ & $218(40)$ \\
\hline Hyperdiploidy & $183(32)$ & $147(80)$ & $36(20)$ & $147(80)$ & $36(20)$ & $147(80)$ & $36(20)$ & $143(78)$ & $40(22)$ \\
\hline $\mathrm{t}(12 ; 21)$ (TEL-AML) & $144(25)$ & $116(81)$ & $28(19)$ & $116(81)$ & $28(19)$ & $116(81)$ & $28(19)$ & $115(80)$ & $29(20)$ \\
\hline $\mathrm{t}(1 ; 19)(\mathrm{E} 2 \mathrm{~A}-\mathrm{PBX})$ & $22(4)$ & $12(55)$ & $10(45)$ & $12(55)$ & $10(45)$ & $12(55)$ & $10(45)$ & $10(45)$ & $12(55)$ \\
\hline No specific lesion & $193(33)$ & $77(40)$ & $116(60)$ & $65(34)$ & $128(66)$ & $65(34)$ & $128(66)$ & $56(29)$ & $137(71)$ \\
\hline Missing & $1(0)$ & $0(0)$ & $1(100)$ & $0(0)$ & $1(100)$ & $0(0)$ & $1(100)$ & $0(0)$ & $1(100)$ \\
\hline MRD & & & & & & & & & \\
\hline Negative & $258(45)$ & $189(73)$ & $69(27)$ & $189(73)$ & $69(27)$ & $185(72)$ & $73(28)$ & $185(72)$ & $73(28)$ \\
\hline Positive & $38(5)$ & $18(47)$ & $20(53)$ & $18(47)$ & $20(53)$ & $16(42)$ & $22(58)$ & $0(0)$ & $38(100)$ \\
\hline Died prior to test & $6(1)$ & $1(17)$ & $5(83)$ & $1(17)$ & $5(83)$ & $1(17)$ & $5(83)$ & $1(17)$ & $5(83)$ \\
\hline Not performed & $277(48)$ & $151(55)$ & $126(45)$ & $139(50)$ & $138(50)$ & $139(50)$ & $138(50)$ & $139(50)$ & $138(50)$ \\
\hline
\end{tabular}

All values represent $\mathrm{N}(\%)$.

$I Q$, Interquartile range; $H R$, High risk; $M R D$, Minimally residual disease; $N$, Number; $S R$, Standard risk; $W B C$, White blood cell.

are often related [17,21]. Algorithms that take cytogenetics into account either directly or indirectly (i.e. through treatment protocol) will therefore reflect a portion of the prognostic ability of MRD. It should also be noted that differences between kappa statistics in this study were small, and may in fact have been due to chance alone.

Though a minority of discrepant cases was due to data entry errors, the majority could be classified into one of three categories. The first group of discordant cases was secondary to the discrepancy between current and past knowledge of disease prognosticators. In ALL for example, new cytogenetic abnormalities associated with poor outcomes continue to be discovered [22,23]. New discoveries take time to be incorporated into clinical practice, such that past children with these risk features may not have had their treatment modified. This resulted in their misclassification as standard risk by the registry-based algorithm.

In the second group, several children had high risk factors that were not specified a priori by the standardized chart review. For example, the inadvertent pretreatment with steroid of children with ALL is widely considered to increase the risk for poor outcome [24]. Despite this, investigators rarely present risk stratification by steroid pretreatment when reporting outcomes, likely due to its relative rarity and inconsistent reporting

Table 4 Measures of agreement between the registry-based algorithm and the biology-based algorithms*

\begin{tabular}{lcccrc}
\hline Biology-based algorithms & Sensitivity & Specificity & PPV & NPV & Kappa, 95 \\
\hline Algorithm 1 (Age, WBC) & 0.95 & 0.88 & 0.83 & 0.97 & $0.80(0.76-0.86)$ \\
Algorithm 2 (Age, WBC, immunophenotype) & 0.95 & 0.91 & 0.88 & 0.97 & $0.85(0.81-0.89)$ \\
Algorithm 3 (Age, WBC, immunophenotype, cytogenetics) & 0.94 & 0.96 & 0.89 & 0.96 & $0.85(0.81-0.90)$ \\
Algorithm 4 (Age, WBC, immunophenotype, cytogenetics, MRD) & 0.90 & 0.93 & 0.91 & 0.92 & $0.83(0.78-0.87)$ \\
\hline
\end{tabular}

*Note that sensitivity, specificity, PPV and NPV were calculated for the ability of the registry-based algorithm to correctly identify high risk patients as defined by the various biology-based algorithms.

$C l$, Confidence interval; MRD, Minimally residual disease; NPV, Negative predictive value; PPV, Positive predictive value; WBC, White blood cell. 
$[10,17,25]$. Such prognosticators are also difficult to obtain, even through chart review. It is worth noting that in the current validation study, treating clinicians were of course aware of these factors and accordingly prescribed higher intensity treatment protocols. Registry-based algorithms incorporating treatment protocol therefore seem to accurately reflect the presence of such prognosticators.

Finally, in the third group, patients met technical standard risk criteria but were nonetheless treated on high risk protocols by their physicians. For example, NCI standard risk criteria are based on the first WBC at the treating institution. In several cases however, when a presenting WBC $<50 \times 10^{9} / \mathrm{L}$ rapidly increased to $\geq 50 \times 10^{9} / \mathrm{L}$ prior to treatment initiation, clinicians chose to base risk stratification on the latter value. Again, treatment protocol functioned as a proxy of physicians' overall impression of disease risk, both when physicians adhered to or deviated from standardized risk criteria. These examples also illustrate how the utility of cancer registries for populationbased research is dependent on the alignment of data capture strategies with clinical applicability [12].

Strengths of this study include the large sample size and the ability to successfully link local data to populationbased registry records. A significant limitation is the ability to generalize our findings to different malignancies and treatment protocols. Some cooperative groups treat ALL with protocols whose names do not differ by risk strata, or where multiple treatment strata are contained within a single protocol name. Osteosarcoma provides another example; though the presence of metastases carries a far worse survival than localized disease, the treatment is generally the same [26]. Treatment-based risk algorithms would therefore not be a valid proxy of disease risk. In addition, some population-based registries may not collect treatment protocol names. Other ways of defining treatment intensity such as the use of specific chemotherapeutic agents or length of treatment have been used but require validation [27]. Even where other registries do collect protocol name, local validation analyses similar to that carried out in this study are likely to still be necessary before further analyses requiring risk stratification can be conducted.

A second limitation concerns the generalizability to other jurisdictions. It is possible that treatment assignment practices vary between institutions, with other centers less likely to use standardized chemotherapy protocols. However, pediatric oncology enjoys a greater degree of standardization than most medical disciplines. Almost all centers treating childhood cancer belong to large cooperative trial groups, and previous research has shown that a majority of cancer patients less that 15 years of age are registered in clinical trials across virtually all metropolitan and rural areas across the United States $[28,29]$. Finally, while the method presented in this paper is a valid way of approximating disease risk, and therefore in predicting survival and relapse-based outcomes, its ability to predict other outcomes (e.g. long-term side effects of treatment) is unknown.

\section{Conclusions}

In conclusion, in this study we found that a registryderived algorithm based on treatment protocol name and age at diagnosis was a valid method of approximating disease risk. Future studies may consider using treatmentbased algorithms for the purpose of risk stratification when conducting registry-based research in pediatric oncology, though validation in other malignancies and using other indicators of treatment intensity is required.

\section{Abbreviations}

ALL: Acute lymphoblastic leukemia; MRD: Minimal residual disease; OCR: Ontario cancer registry; POGONIS: Pediatric oncology group of ontario networked information system; WBC: White blood cell.

\section{Competing interests}

The authors declared that they have no competing interest.

\section{Authors' contributions}

SG conducted the chart review and performed the analyses. All authors participated in the design of the study and helped draft the manuscript. All authors read and approved the final manuscript.

\section{Acknowledgements}

SG is supported by a Doctoral Fellowship from the Canadian Institutes for Health Research. LS is supported by a New Investigator Award from the Canadian Institutes of Health Research.

\section{Author details}

${ }^{1}$ Division of Haematology/Oncology and Program in Child Health Evaluative Sciences, Hospital for Sick Children, 555 University Avenue, Toronto, Ontario, Canada M5G 1X8. ${ }^{2}$ Pediatric Oncology Group of Ontario, 480 University Avenue, Suite 1014, Toronto, Ontario, Canada M5G 1V2. Institute for Clinical Evaluative Sciences, G1 06, 2075 Bayview Avenue, Toronto, Ontario, Canada M4N 3M5. ${ }^{4}$ Division of Paediatric Medicine, Hospital for Sick Children, 555 University Avenue, Toronto, Ontario, Canada M5G 1X8. ${ }^{5}$ Department of Paediatrics and Institute for Health, Policy Management and Evaluation, University of Toronto, 155 College Street, Suite 425, Toronto, Ontario, Canada M5T 3 M6.

Received: 19 November 2012 Accepted: 21 May 2013

Published: 30 May 2013

\section{References}

1. Ellison LF, Pogany L, Mery LS: Childhood and adolescent cancer survival: a period analysis of data from the Canadian Cancer Registry. Eur J Cancer 2007, 43:1967-1975.

2. Pui C-H, Pei D, Pappo AS, Howard SC, Cheng C, Sandlund JT, Furman WL, Ribeiro RC, Spunt SL, Rubnitz JE, et al: Treatment outcomes in black and white children with cancer: results from the SEER database and St. Jude Children's Research Hospital, 1992 through 2007. J Clin Oncol 2012, 30:2005-2012.

3. Smith MA, Seibel NL, Altekruse SF, Ries LAG, Melbert DL, O'Leary M, Smith FO, Reaman GH: Outcomes for children and adolescents with cancer: Challenges for the twenty-first century. J Clin Oncol 2010, 28:2625-2634.

4. Hunger SP, Lu X, Devidas M, Camitta BM, Gaynon PS, Winick NJ, Reaman $\mathrm{GH}$, Carroll WL: Improved survivial for children and adolescents with acute lymphoblastic leukemia between 1990 and 2005: a report from the Children's Oncology Group. J Clin Oncol 2012, 30:1663-1669.

5. Aplenc R, Fisher BT, Huang YS, Li Y, Alonzo TA, Gerbing RB, Hall M, Bertoch $D$, Keren $R$, Seif $A E$, et al: Merging of the national cancer institute-funded cooperative oncology group data with an administrative data source to 
develop a more effective platform for clinical trial analysis and comparitive effectiveness research: a report from the Children's oncology group. Pharmacoepidemiol Drug Saf 2012, 21(Suppl 2):37-43.

6. Walker DM, Fisher BT, Seif AE, Huang YS, Torp K, Li Y, Aplenc R: Dexrazoxane use in pediatric patients with acute lymphoblastic or myeloid leukemia from 1999 and 2009: analysis of a national cohort of patients in the pediatric health information systems database. Pediatr Blood Cancer 2012, 60(4):616-620.

7. Moller TR, Garwicz S, Barlow L, Winther JF, Glattre E, Olafsdottir G, Olsen JH, Perfekt R, Ritvanen A, Sankila $R$, et al: Decreasing late mortality among five-year survivors of cancer in childhood and adolescence: a population-based study in the Nordic Countries. J Clin Oncol 2001, 19(13):3173-3181

8. Fisher BT, Zaoutis TE, Leckerman KH, Localio R, Aplenc R: Risk factors for renal failure in pediatric patients with acute myeloid leukemia: a retrospective cohort study. Pediatr Blood Cancer 2010, 55(4):655-661.

9. Koch SV, Kejs AMT, Engholm G, Johansen C, Schmiegelow K: Educational attainment among survivors of childhood cancer: A population-based cohort study in Denmark. Br J Cancer 2004, 91(5):923-928.

10. Pui CH, Campana D, Pei D, Bowman WP, Sandlund JT, Kaste SC, Ribeiro RC, Rubnitz JE, Raimondi SC, Onciu M, et al: Treating childhood acute lymphoblastic leukemia without cranial irradiation. N Engl J Med 2009, 360:2730-2741.

11. Maris JM: Recent advances in neuroblastoma. N Engl J Med 2010, 362:2202-2211.

12. Meyer AM, Carpenter WR, Abernethy AP, Sturmer T, Kosorok MR: Data for cancer comparative effectiveness research: Past, present, and future potential. Cancer 2012, 118:5186-5197.

13. Rubnitz JE, Inaba H, Dahl G, Ribeiro RC, Bowman WP, Taub J, Pounds S, Razzouk BI, Lacayo NJ, Cao X, et al: Minimal residual disease-directed therapy for childhood acute myeloid leukemia: Results of the AML02 multicenter trial. Lancet Oncol 2010, 11(6):543-552.

14. Greenberg ML, Barr RD, DiMonte B, McLaughlin E, Greenberg C: Childhood cancer registries in Ontario, Canada: lessons learned from a comparison of two registries. Int J Cancer 2003, 105(1):88-91.

15. Arico M, Schrappe M, Hunger SP, Carroll WL, Conter V, Galimberti S, Manabe A, Saha V, Baruchel A, Vettenranta K, et al: Clinical outcome of children with newly diagnosed Philadelphia chromosome-positive acute lymphoblastic leukemia treated between 1995 and 2005. J Clin Oncol 2010, 28(31):4755-4761.

16. Heerema NA, Nachman JB, Sather HN, Sensel MG, Lee MK, Hutchinson $R$, Lange BJ, Steinherz PG, Bostrom B, Gaynon PS, et al: Hypodiploidy with less than 45 chromosomes confers adverse risk in childhood acute lymphoblastic leukemia: a report from the children's cancer group. Blood 1999, 94(12):4036-4045.

17. Borowitz MJ, Devidas M, Hunger SP, Bowman WP, Carroll A, Carroll WL, Linda S, Martin PL, Pullen J, Viswanatha D, et al: Clinical significance of minimal residual disease in childhood acute lymphoblastic leukemia and its relationship to other prognostic factors: a children's oncology group study. Blood 2008, 111:5477-5485.

18. Schrappe M, Camitta B, Pui CH: Long-term results of large prospective trials in childhood acute lymphoblastic leukemia. Leukemia 2000, 14(12):2193-2194.

19. Landis R, Koch GG: The measurement of observer agreement for categorical data. Biometrics 1977, 33(1):159-174.

20. Fleiss JL: Statistical methods for rates and proportions. 2nd edition. New York: John Wiley; 1981.

21. Campana D: Minimal residual disease in acute lymphoblastic leukemia. Hematology 2010, 2010(1):7-12

22. Chen I-M, Harvey RC, Mullighan CG, Gastier-Foster F, Wharton W, Kang H, Borowitz MJ, Camitta BM, Carroll AJ, Devidas M, et al: Outcome modeling with CRLF2, IKZF1, JAK, and minimal residual disease in pediatric acute lymphoblastic leukemia: a children's oncology group. Blood 2012, 119(15):3512-3522.

23. Moorman AV, Richards SM, Robinson HM, Strefford JC, Gibson BES, Kinsey SE, Eden TOB, Vora AJ, Mitchell CD, Harrison CJ: Prognosis of children with acute lymphoblastic leukemia (ALL) and intrachromosomal amplification of chromosome 21 (iAMP21). Blood 2007, 109(6):2327-2330.

24. Revesz T, Kardos G, Kajtar P, Schuler D: The adverse effect of prolonged prednisolone pretreatment in children with acute lymphoblastic leukemia. Cancer 1985, 55(8):1637-1640.
25. Bonilla M, Gupta S, Vasquez R, Fuentes SL, DeReyes G, Ribeiro KCB, Sung L: Predictors of outcome and methodological issues in children with acute lymphoblastic leukemia in El Salvador. Eur J Cancer 2010, 46(18):3280-3286.

26. Arndt CA, Rose PS, Folpe AL, Laack NN: Common musculoskeletal tumors of childhood and adolescence. Mayo Clin Proc 2012, 87(5):475-487.

27. Fisher BT, Harris T, Torp K, Seif AE, Shah A, Huang YS, Bailey LC, Kersun LS, Reilly AF, Rheingold SR, et al: Establishment of an 11-year cohort of 8733 pediatric patients hospitalized at United States free-standing children's hospitals with de novo acute lymphoblastic leukemia from health care administrative data. Med Care 2012 [Epub ahead of print].

28. Bleyer A, Budd T, Montello M: Adolescents and young adults with cancer: the scope of the problem and criticality of clinical trials. Cancer 2006, 7(Suppl):1645-1655.

29. Bleyer A: The adolescent gap in cancer treatment. J Regist Manage 1996 23:114-115.

doi:10.1186/1471-2288-13-68

Cite this article as: Gupta et al:: Validation of a registry-derived risk algorithm based on treatment protocol as a proxy for disease risk in childhood acute lymphoblastic leukemia. BMC Medical Research Methodology 2013 13:68.

\section{Submit your next manuscript to BioMed Central and take full advantage of:}

- Convenient online submission

- Thorough peer review

- No space constraints or color figure charges

- Immediate publication on acceptance

- Inclusion in PubMed, CAS, Scopus and Google Scholar

- Research which is freely available for redistribution 